\title{
Collision Reduction in Cognitive Radio using Multichannel 1-persistent CSMA combined with Reinforcement Learning
}

\author{
Haibin Li, David Grace, Paul D. Mitchell \\ Department of Electronics \\ The University of York \\ York, United Kingdom \\ $\{$ h1527|dg|pdm106\}@ohm.york.ac.uk
}

\begin{abstract}
In this paper a novel multiple access scheme, MCSMA-RL, is proposed for secondary users which combines multichannel 1-persistent CSMA and reinforcement learning. The scheme effectively reduces the probability of packet collisions among primary and secondary users sharing common spectrum. Compared with multichannel CSMA without learning, the throughput and packet loss of M-CSMA-RL shows a significant improvement in a distributed cognitive radio scenario in situations where primary users operate with TDMA/FDMA. The results show how the M-CSMA-RL scheme improves both primary and secondary user's throughput at various offered traffic levels and with different ratios of primary and secondary user offered traffic.
\end{abstract}

Keywords- Cognitive Radio; Multiple Access Schemes; Channel Assignment; Multichannel CSMA; Reinforcement Learning

\section{INTRODUCTION}

Cognitive Radio (CR) [1, 2] promises to improve spectrum utilization by supporting secondary users (SU) that are aware of radio spectrum holes, enabling them to intelligently share the spectrum with primary users (PU). The PU has top priority to use the specified spectrum which has been licensed to them. The SU needs to be able to explore and exploit idle spectrum which is not being used by primary and other secondary users, with the ability to access the idle spectrum without causing harmful interference to PU. Accordingly, when the PU and SU share the same spectrum, control and reduction of the interference is one of the crucial issues for both the PU and SU to maintain a high quality of service $(\mathrm{QoS})$ in a cognitive radio network [3]. Furthermore, from channel capacity and throughput perspective, the interaction from secondary users can be considered as one of the most important sources of interference that causes data packet collisions, thus interrupting the transmission link of both primary and secondary users [4]. Consequently, in order to improve the link maintenance for both primary and secondary users, a novel spectrum sharing protocol is proposed for the SU which combines multichannel 1-persistent carrier sense multiple access (M-1p-CSMA) [5, 6] and Reinforcement Learning (RL) [7], enhancing secondary users awareness of the radio environment, and enabling them to intelligently avoid packet collisions. The combination scheme is named M-CSMA-RL.

The 1-persistent CSMA protocol is designed to maximize channel utilization and achieve optimum throughput by never letting the channel become idle if some users are waiting for transmission [5]. However, in the situation when more than one user is waiting for transmission, all users will transmit with a probability of 1 when the channel becomes idle, thus leading to a conflict, which will also occur with probability of 1. The M-1p-CSMA protocol [6] is an extension from the single channel scheme that allows users to transmit on different channels at the same time, thus reducing the probability of conflict compared with the single channel case. A user of M-1p-CSMA verifies the absence of transmissions from other users by relying on the carrier sensing function, and then is allowed to immediately transmit a ready data packet as soon as a channel is detected as available. From a cognitive radio based secondary user perspective, the characteristics of M-1p-CSMA provide an excellent way to access the idle spectrum when no primary user is transmitting. It again improves the spectrum utilization compared with other retransmission techniques, since data can be transmitted with probability of 1 in idle channels, without an unnecessary wait. Nonetheless, when primary and secondary users share the same spectrum, it is also very difficult to fully ensure a conflict free transmission due to the fact that:

- $\quad$ two or more CSMA users may become ready during a transmission when all channels are fully occupied, and when a channel becomes idle they will both transmit simultaneously, resulting in a collision. Such collisions will keep occurring with probability of one, the same as the single channel case.

- A carrier may not be sensed because of the propagation delay, thus a CSMA user with a ready data packet may transmit during the transmission period of other users.

- Primary users do not perceive the existence of secondary users, and a collision occurs when the PU transmits packets during SU transmissions.

In order to prevent collisions occurring when there are hidden terminals, previous studies have proposed a cognitive MAC with CSMA/CA (Collision Avoidance) [8, 9] for secondary users. In [8], the authors proposed intermittent overlay CSMA/CA scheme to control the collisions using a RTS and CTS exchange mechanism. Secondary users will obtain better information of whether other users are transmitting or not, thus preventing collisions from occurring. Furthermore, another cognitive radio MAC is proposed using CSMA/CA in [9]. This 
protocol introduces a dual sensing algorithm with outband sensing to exploit idle spectrum, and inband sensing to detect PU transmissions. A MAC-controller keeps updating the information and prevents collisions among the primary and secondary users

Different from the CSMA/CA approach to prevent collisions amongst primary and secondary users, M-CSMA$\mathrm{RL}$ allows the SU to intelligently and autonomously make a decision by choosing an idle channel to access without wholly relying on central control to avoid interference, thus increasing robustness and spectrum efficiency in a distributed spectrum sharing environment. In this context, reinforcement learning [7] is applied to the SU as an approach to implement selforganizing procedures, and avoid unnecessary interaction amongst primary and secondary users. In previous research, RL has shown inherent cognitive capabilities by maximizing a numerical reward from a continuous interaction with a radio environment [10].

In order to examine the performance of the secondary users by operating M-CSMA-RL, it is applied to a distributed cognitive radio network where primary users operate with a TDMA/FDMA system [11]. The TDMA/FDMA is a conventional contention free transmission scheme used similar to that used in the GSM cellular network, for example [12]. During a transmission, users can only use the designed time slots on a channel. The purpose of this paper is to explain the principle and the processing procedure of the M-CSMA-RL protocol in this proposed cognitive radio network, and examine its performance from the packet interaction, system capacity and SU throughput perspectives.

The rest of paper is organized as follows. Section II introduces the cognitive radio model and its assumptions for the supporting simulation-based analysis. Section III explains the details of the M-CSMA-RL scheme, including the principle of M-1p-CSMA and reinforcement learning, analysis of the collision mechanisms in the scenario, and the algorithmic procedure of the M-CSMA-RL scheme. Section IV provides the simulation results and analysis based on a throughput comparison between M-1p-CSMA and M-CSMARL when working with a primary network, the PU packet loss rate when SU shares the spectrum, and the process of reinforcement learning. Finally, a conclusion is given in section $\mathrm{V}$.

\section{SCENARIO MODEL AND SYSTEM ASSUMPTIONS}

In order to examine the improvement of link maintenance by applying M-CSMA-RL to secondary users, a cognitive radio scenario is built based on a radio network with primary users using TDMA/FDMA. The scenario models the uplink transmissions of primary and secondary users operating in a common cell of a multichannel cellular network. Each type of user transmits data packets to their own receiver, as shown in figure 1 . We assume all secondary users can hear each other, i.e. there are no hidden terminals. Collisions will only occur as a result of primary and secondary user interaction, including as a result of the vulnerable period in CSMA.

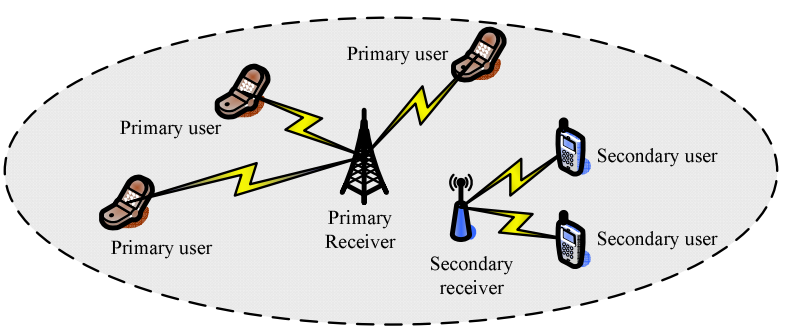

Figure 1. Cognitive Radio Senario Model

During the transmission, primary users have top priority to transmit data packets using their designated time slots on a specific channel, and they do not perceive the existence of secondary users. As a secondary user in the CR model, each entity contains a reinforcement learning (RL) engine that acquires its transmission experience as a reward value to adjust the next transmission.

In the scenario, for better analysis of the interaction amongst primary and secondary users, we assume that transmissions are successful or suffer a collision. We will not explicitly consider the signal to noise ratio, and assume that in the absence of collisions all channels are error free. Furthermore, we define the data traffic as Poisson distributed [13] at both primary and secondary receivers, with a mean packet arrival rate $\tau$ packets/second. The value of $\tau$ varies according to different levels of offered traffic. It is also assumed that the packet transmission time is a constant $T$, as all packets are constant length. Other parameters of the cognitive radio network are shown in table 1 .

TABLE I. NETWORK PARAMETERS

\begin{tabular}{|c|c|}
\hline Parameters & Values \\
\hline Number of channels ' $M$ ' & 10 \\
\hline Bandwidth per channel, ' $B$ ' & $128 \mathrm{KHz}$ \\
\hline Modulation Scheme & QPSK \\
\hline Bit rate per channel, ' $r$ ' & $512 \mathrm{kbits} / \mathrm{s}$ \\
\hline Packet length, ' $l$ ' & $128 \mathrm{bits}$ \\
\hline Packet transmission time ' $T$ ' & $0.25 \mathrm{e}^{-3}$ \\
\hline $\begin{array}{c}\text { Number of Primary Users, ' } N_{P}{ }^{-3} \\
\text { Number of Secondary Users, ' } N_{S} \text { ' }\end{array}$ & 100 \\
\hline $\begin{array}{c}\text { Secondary user sensing and transmission } \\
\text { normalized delay ' } a \text { ' }\end{array}$ & 0.05 \\
\hline
\end{tabular}

\section{M-CSMA-RL PROTOCOL}

The use of multi-channels will reduce the probability of collision between two users, as they may assign themselves different channels to transmit on. Generally, there are two types of channel selection schemes for multichannel CSMA, Random Choice (RC) and Idle Choice (IC) [6]. The user with random choice randomly chooses a channel before sensing. The user with Idle Choice senses all channels first and randomly chooses a channel amongst those that are sensed idle. Compared with the idle choice, a drawback of multichannel random choice is that packets may not be transmitted while some channels are idle. Therefore, we will apply the idle choice as the basic channel assignment scheme. 


\section{A. Multichannel 1-persistent CSMA and its modification}

The extension to M-1p-CSMA provides a solution to reduce the probability of collision to some extent. However, according to the transmission method of single channel $1 \mathrm{p}$ CSMA, a drawback of M-1p-CSMA is that collisions will also occur with a probability of one if two or more CSMA users become ready in a situation where all channels are fully occupied. In a cognitive radio scenario, this drawback could heavily affect the transmission quality of secondary users. In order to address this problem in the M-CSMA-RL scheme, the transmission procedure of M-1p-CSMA is modified as:

1) A user with a ready packet senses all channels first. If more than one channel is sensed idle, the user will randomly choose one from those that are sensed idle.

2) If the transmitted packets collide, the user will immediately sense all channels again, and retransmit the packet on a randomly chosen idle channel.

3) If all channels are sensed busy, the user will retransmit the packet at some later time on a free channel according to a random back off interval with a mean value of $\lambda$.

By applying the back off interval, users will mostly avoid each other by retransmitting the packet at different times instead of transmitting at the same time and colliding with each other with a probability of 1 .

\section{B. Collision situations among PU and $S U$}

Collisions among primary and secondary users are an important packet interaction that affects the transmission link of both primary and secondary users in a cognitive radio network [4]. To avoid the interaction, firstly, we have to understand the collision mechanism from both the primary and secondary user's perspective in the situation of M-1p-CSMA without applying RL.

1) From the PU transmission perspective in figure 2, due to the fact that the PU is not aware of the SU transmission, a collision occurs with the PU packet when:

a) an $S U$ packet has been transmitted at a transmission time ' $T$ ' prior to the PU starting to transmit a packet

b) an SU transmits a packet during the first vulnerable period ' $a$ ' [5], which is defined as the transmission delay of the PU packet.

2) From the SU transmission perspective in figure 3, a collision occurs with the SU packet when:

a) an SU or PU packet is transmitted during the first vulnerable period ' $a$ ' of the transmitting $S U$ packet.

b) a PU packet is transmitted during the transmission time of an SU transmission.



Figure 2. The collision situation during a PU transmission

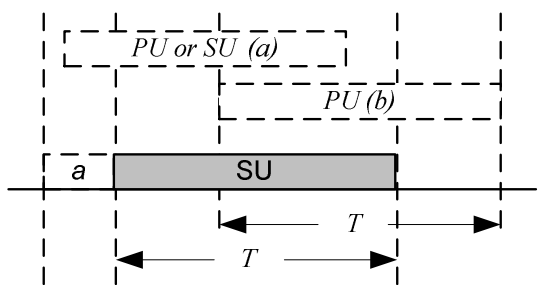

Figure 3. The collision situation during a SU transmission

To conclude, in order to ensure that both primary and secondary packets are successfully transmitted in this cognitive radio network, we need to ensure that

1) No SU packet is transmitted in the vulnerable period of ' $T+a$ ' before the time that a PU starts its transmission.

2) No PU or other SU transmits a packet during the first ' $a$ ' vulnerable period of a SU transmisison.

Due to the fact that the primary users have priority use of the spectrum, and its transmission should not be interrupted by any secondary users in a cognitive radio network, we can only limit the transmission of secondary users by trying to reduce the probability that two or more users will transmit simultaneously in the vulnerable period of their transmissions, and avoid the primary user's packets.

\section{Applying Reinforcement Learning to $S U$}

In order to avoid collisions among the primary and secondary users, we apply the RL algorithm in secondary users to make them aware of the radio environment, and intelligently assign a channel with the best chance of successful packet transmission by considering the previous experience on each channel. As an interaction with the radio environment, the current transmission information of each channel will also be updated for the next channel assignment. The RL algorithm assures each secondary user is assigned an optimum channel through maximization of an average reward over the long-term [14]. More precisely, consider a cognitive radio network with $M$ available channels, $N_{P}$ primary users and $N_{S}$ secondary users. Each secondary user is defined to have two reward weight vectors associated with each channel, which are the channel sensing weight ' $W_{S}$ ' and packet transmission weight ' $W_{T x}$ ' as shown below

$W_{S \_k}=\left\{W_{S \_k l}, W_{S \_k 2} \ldots W_{S \_k m}\right\}$ and

$W_{T x_{-} k}=\left\{W_{T x_{-} k l}, W_{T x_{-} 22} \ldots W_{T x_{-} k m}\right\}, k \in\left\{1, N_{S}\right\}, m \in\{1, M\}$

The value of the sensing weights $W_{S}$ and transmission weights $W_{T x}$ depend on the reward factors from the sensing and transmission results, which are $\Delta f_{S}$ and $\triangle f_{T x}$ respectively. The weight value of each channel will be adjusted by those reward factors after every transmission. Accordingly, the weight vectors of the $k$-th secondary user on the $m$-th channel for the next iteration can be expressed as

$$
\begin{aligned}
& W_{S_{-} k m}(t+1)=W_{S_{-} k m}(t)+\Delta f_{S}(t) \\
& W_{T_{x_{-} k m}}(t+1)=W_{T_{x_{-} k m}}(t)+\Delta f_{T x}(t)
\end{aligned}
$$

The procedure of the reward function is

1) For the spectrum sensing weight of every transmission $t$, the sensing factor $\triangle f_{S}$ will be rewarded with a value of 1 if 
the channel is sensed idle. On the other hand, the factor will be rewarded with a value of -1 if the channel is sensed busy.

2) For the transmission period of every learning step $t$, if the transmission succeeds on the channel, the transmission factor $\triangle f_{T x}$ on that channel will be rewarded with a value of 1 . If the transmission fails, the transmission factor on that channel will be rewarded -1 .

During a transmission of the $k$-th secondary user, the user will use the information of the experienced transmissions by comparing the weight vectors of each channel, and then will make a channel selection decision by choosing the idle channel with the highest average value of the $W_{S_{-} k}$ and $W_{T x_{-} k}$. In the situation that ' $i$ ' channels sensed idle, where $i \leq M$. we have

$$
W_{k_{-} \max }=\max \left\{\frac{1}{2}\left\{W_{S_{-} k i}+W_{T_{x_{-}} k i}\right\}\right\}
$$

$W_{k \text { max }}$ is the maximum average value of the sensing vector and transmission vector. $W_{S k i}$ and $W_{T x k i}$ are the sensing and transmission vectors of idle channels respectively. The idle channel with the $W_{k \text { max }}$ is chosen for the transmission. To set the initial value of each weight factor, the first data packet of the $k$-th secondary user is randomly transmitted on an idle channel from those that are sensed idle.

\section{M-CSMA-RL algorithm}

An overview of the M-CSMA-RL algorithm is shown in figure 4, which describes the transmission procedure of a secondary user when sharing the same spectrum with primary users. In general, the processing can be summarized as:

1) An SU with a new or retransmission packet ready to transmit.

2) In terms of interaction with the radio environment, the $S U$ senses all channels before transmission. The sensing weight $W_{S_{-} k}$ and idle channels are updated in the Learning and Reasoning Engine. If there is no channel sensed idle, the packet transmission will randomly backoff for an interval with a mean value of $\lambda$.

3) A decision will be made by considering the experience, encapsulated in the transmission weights $W_{S_{-} k}$ and $W_{T x_{-} k}$ on each idle channel. The idle channel with highest average weight will be accessed.

4) After the transmission period, the transmission weight $W_{T x}$ will be updated in the Learning and Reasoning Engine. If the packet is successfully transmitted, the SU will process the next packet. Otherwise, the failed packet will be retransmitted immediately.

\section{SIMULATION RESULTS AND ANALYSIS}

In this paper, an event based simulation based on a Monte Carlo method [15] is used to examine the performance of MCSMA-RL by applying a large number of random trials (data packets) to compute statistical results. The sensing period is assumed to be neglectable.

Figure 5 shows the throughput results of primary and secondary users in the cognitive radio model when they have the same offered traffic level. Two different schemes are applied to the secondary users, one is M-1p-CSMA, and the other is M-CSMA-RL. Primary users operate with pure TDMA/FDMA.

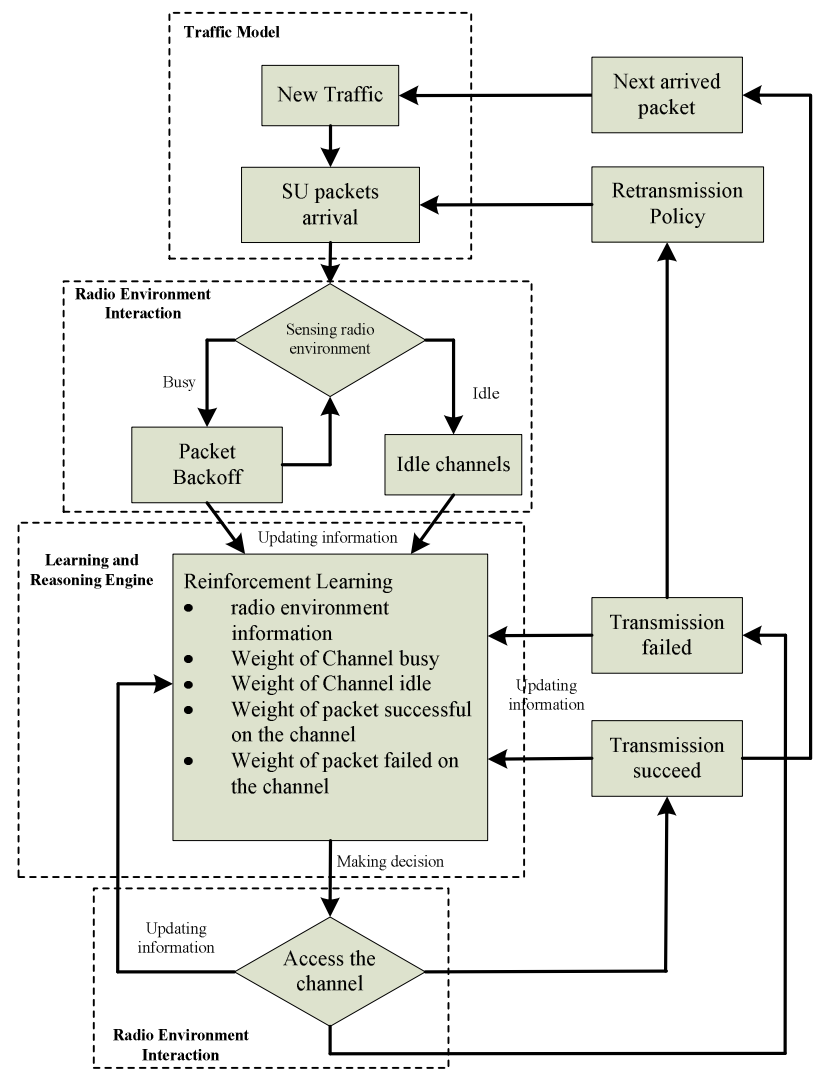

Figure 4. Flow chart of the M-CSMA-RL algorithm

The performance of the two secondary user schemes, with and without RL can be compared. It can be seen that the throughput of both primary and secondary users show better performance in the mode with M-CSMA-RL. The throughput loss of primary users compared with pure TDMA/FDMA has been reduced significantly. Regarding the improvement rate, the throughput of the secondary user shows a greater increase in the model with RL than that of the primary user. This is because secondary users have the probability to collide with both primary and secondary users, and the primary users collide with just secondary users. By applying RL, the secondary users not only avoid collisions with primary users, but also other secondary users, thus providing them with a greater throughput improvement. Furthermore, as the primary user's traffic increases, the amount of idle spectrum is decreased. Consequently, the throughput of secondary users will be reduced to zero when the throughput of primary users achieves the value of 1 , which means the spectrum is fully occupied by primary users.

Figure 6 shows the packet loss of primary and secondary users in the CR model with and without RL. The packets loss is defined as the number of collision packets dividing by the number of total transmitted packets. The results are obtained at various secondary user's offered traffic to total offered traffic ratios $\rho$. A $\rho$ value equal to 0.1 represents $10 \%$ secondary user's traffic and $90 \%$ primary user's traffic in the system. According to figure 6 , the total offered traffic is set to the 0.7 Erlangs per channel, which is the level when the secondary user achieves peak throughput value. The packets loss is calculated as the percentage of colliding packets to the total 
number of packets that have been transmitted. The higher the traffic ratio of the users, the lower packet loss. Comparing the two different schemes, the packet losses of both primary and secondary users are reduced significantly by applying $\mathrm{M}$ CSMA-RL at every ratio. More importantly, the packet loss of primary users has been limited to less than $10 \%$ for all traffic ratios.

These results confirm that the secondary users with MCSMA-RL can effectively control the data packet interaction to primary users, reducing the collision amongst different users, and improving the quality of the transmission link for both primary and secondary users through increased experience obtained through SU learning of packet transmissions.

Figure 7 illustrates the learning process of M-CSMA-RL during a secondary user's transmission in the situation where $\rho$ is equal to 0.5 and the total offered traffic is 0.7 Erlangs per channel. Each iteration number includes 10 data packets of the SU. It clearly shows the throughput increases during the learning process, and finally achieves a stable level. It is likely this reinforcement learning technique could be incorporated into more sophisticated collision avoidance schemes, such as CSMA/CA, in a frequency reuse scenarios. This would provide further improvement to the collision performance in these situations.

\section{CONCLUSION}

In this paper, a multichannel 1-persisitent CSMA scheme combined with reinforcement learning is proposed for secondary users in cognitive radio. The sensing function of CSMA enables secondary users to be aware of the idle spectrum, and the reinforcement learning enables secondary users to intelligently share the spectrum with primary users by considering their experienced transmission, and choosing the channel with more successful transmission probability.

The M-CSMA-RL scheme is applied to secondary users in a distributed cognitive radio model based on primary users operating with a TDMA/FDMA system. The results show that the throughput of both primary and secondary users are improved compared with the scheme without learning, which implies that M-CSMA-RL can effectively reduce packet collisions for both primary and other secondary users, thereby improving the probability of successful transmissions for both primary and secondary users.

\section{REFERENCES}

[1] J. Mitola, and G. Maguire, Cognitive Radio: Making Software Radios More Personal. IEEE Personal Communications 1999.

[2] L. Doyle, Essentials of Cognitive Radio. The Cambridge Wireless Essentials Series. 2009. Cambridge University Press. 233.

[3] K. Chen, and R. Prasad, Cognitive Radio Networks. 2009. John Wiley \& Sons Ltd. 359.

[4] H. Li, D. Grace, P. Mitchell, Throughput Analysis of Non-persistent CSMA Combined with TDMA and its implication for Cognitive Radio, Journal on IET Communications, 2010, accepted for publication.

[5] L. Kleinrock, and F.A. Tobagi, Packet Switching in Radio Channels: Part 1 - Carrier Sense Multiple-Access Modes and Their ThroughputDelay Characteristics. IEEE Transactions on Communications, 1975. COM-23(12).

[6] M. A. Marsan, and D. Roffinella, Multichannel Local Area Network Protocols. IEEE Journal on Selected Areas in Communications, 1983. $\mathrm{SAC}-1(5)$

[7] L. P. Kaelbling, M.L. Littman, and A.W. Moore, Reinforcement Learning: A Survey. Journal of Artificial Intelligence Research, 1996. 4(1996): p. 237-285.

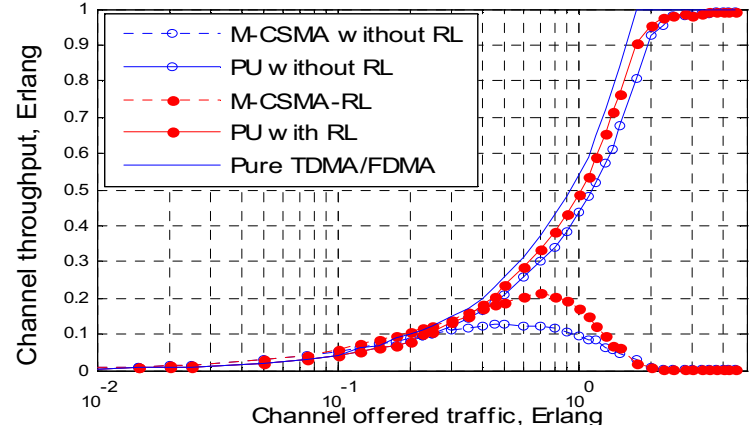

Figure 5. Throughput Comparison $\mathrm{M}=10, \mathrm{SU}$ to total traffic ratio 0.5

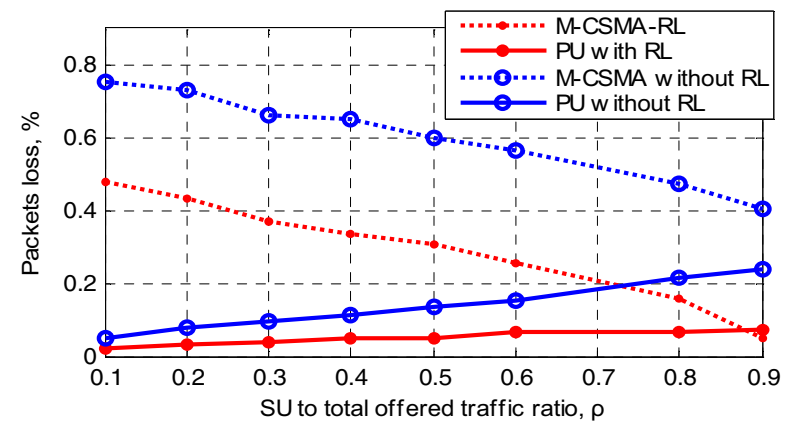

Figure 6. Packets Loss of PU and SU

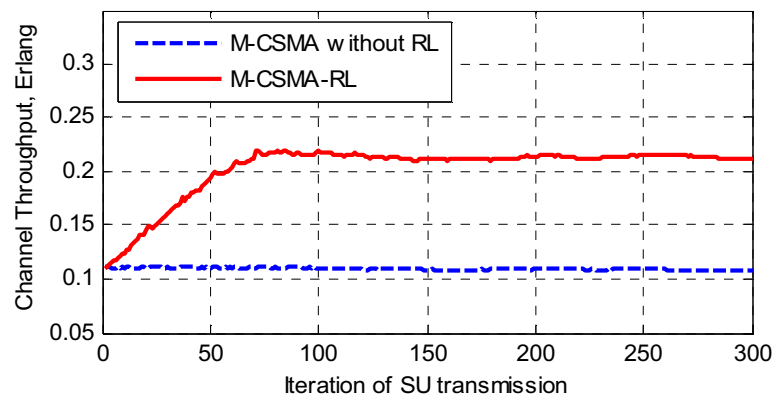

Figure 7. Processing of Reinforcement Learning

[8] A. V. Adamis, K.N. Maliatsos, and P. Constantinou. Methods for Reducing Interference caused to Licensed Systems by OverlayCSMA/CA Cognitive Radios. in Cognitive Radio Oriented Wireless Networks and Communications, 3rd International Conference on CrownCom. 2008. Singapore.

[9] Q. Zhang, F. Fitzek, and V. Iversen. Cognitive Radio MAC protocol for WLAN. in IEEE 19th International Symposium on Personal, Indoor and Mobile Radio Communications. 2008. Cannes.

[10] F. Bernardo, et al. Distributed Spectrum Management based on Reinforcement Learning. in Cognitive Radio Oriented Wireless Networks and Communications, CROWNCOM 09'. 2009. Hannover.

[11] M. Rahnema, Overview of the GSM System and Protocol Architecture, in IEEE Communications Magazine. 1993. p. 92-100.

[12] M. Mouly, and M.-B. Pautet, The GSM System for Mobile Communications. 1992, Telecom Publishing. 701.

[13] P. A. Moran, An Introduction to Probability Theory. 1984, Oxford University Press. 524.

[14] T. Jiang, D. Grace, Y. Liu, Performance of Cognitive Radio Reinforcement Spectrum Sharing Using Different Weighting Factors, Communications and Networking in China, Third International Conference on ChinaCom. 2008.

[15] M. H. Kalos, and P.A. Whitlock, Monte Carlo Methods. 1986, John Wiley \& Sons. 208. 